\title{
Are reinforcement sensitivity personality constructs and attentional control important predictors of restrictive disordered eating?
}

\author{
Andrew R. du Rocher, Jessica Barker, Monika I. Chalupka, Anna France, Raisa S. Habib, Joel H. Holzer, \\ Bethany M. R. Johnston, Heather Mee, Imaan Mohammed, \& Rebecca Quail
}

Department of Psychology, Sociology and Politics, Sheffield Hallam University

\begin{abstract}
The revised Reinforcement Sensitivity Theory (rRST) perspective on personality suggests that a neuropsychological behavioral inhibition system (BIS), behavioral approach system (BAS), and a fight-flight-freeze system (FFFS) produce the key personality traits involved in approach and avoidance behaviors. This perspective on personality can be used as a framework for understanding psychopathology. Self-report research on $r R S T$ suggests that restrictive disordered eating relates to elevated BIS sensitivity, elevated FFFS sensitivity, and possibly dysfunctional BAS sensitivity. Disordered eating can also relate to reduced trait mindfulness, which is a broadly defined construct. Trait mindfulness is positively correlated with attentional control (AC) which is a more specific component of our cognitive architecture that incorporates attentional focusing and attentional shifting processes. It is unknown how BIS and AC interact to predict restrictive disordered eating. We tested how self-reported BIS, BAS, and FFFS sensitivity, AC, and trait mindfulness relate to restrictive disordered eating in 464 healthy participants, and 177 participants with a history of psychiatric disorder. We provide new evidence that elevated restrictive disordered eating relates to reduced self-reported $A C$ abilities (in addition to elevated BIS, and elevated FFFS sensitivity). We illustrate that the combination of high BIS and low AC predicts high levels of restrictive disordered eating (but not in all participants), whereas low BIS and high AC predicts lower levels of restrictive disordered eating (but not in all participants). We discuss how understanding the relationship between personality and AC can inform the design of future intervention studies.
\end{abstract}

Keywords: personality; restrictive disordered eating; reinforcement sensitivity, attentional control; mindfulness

Revised Reinforcement Sensitivity Theory (rRST; Gray \& McNaughton, 2000) suggests that individual differences in sensitivity of neuropsychological systems involved in motivation and emotion produce the key personality constructs involved in approach and avoidance behaviors. The perspective of rRST provides a biological explanation of personality that can be used as a framework for understanding affective disorders, substance use disorders, and eating disorders (Bijttebier, Beck, Claes, \& Vandereycken, 2009). Disordered eating refers to a spectrum of problematic eating behaviors including unhealthy dieting, restrictive eating, and binge-purge behaviors, and it is a risk factor for the development of eating disorders, which often co-occur with anxiety and/or depression (Pereira \& Alvarenga, 2007), and also impaired attentional control (AC; Roberts et al., 2007). Longitudinal research shows that disordered eating predicts poor mental health at a ten year follow up stage (Kärkkäinen et al., 2018). In the present study we examine the relationship between rRST defined personality constructs, AC, and restrictive disordered eating in the general population.

The rRST (Gray \& McNaughton, 2000) perspective on personality includes a behavioral inhibition system (BIS), a behavioral approach system (BAS), and a fight-flight-freeze

Correspondence concerning this article should be addressed to Dr. Andrew R. du Rocher, Centre for Behavioral Science and Applied Psychology; Department of Psychology, Sociology and Politics. Sheffield Hallam University, Collegiate Crescent, Broomhall, Sheffield, South Yorkshire, S10 2BP UK. E-mail: andy.r.du.rocher@gmail.com or ad7910@exchange.shu.ac.uk system (FFFS). In rRST, the BAS facilitates approach, consummatory, and anticipated reward behaviors (Gray \& McNaughton, 2000), and it theoretically underlies the trait of extroversion (Smillie, 2008). The FFFS facilitates fearful avoidance responses to aversive stimuli. The BIS generates anxiety when the BAS and the FFFS are co-activated, and it resolves motivational conflict. Dispositional elevated BIS sensitivity increases the likelihood of an FFFS response (Gray \& McNaughton, 2000). Theoretically, both BIS and FFFS sensitivity underlie the trait of neuroticism (Smillie, 2008). Phenotypical levels of BIS, BAS, and FFFS sensitivity can be measured using self-report personality questionnaires. For example, restrictive disordered eating in anorexia is related to greater self-reported BIS and FFFS sensitivity, and to reduced self-reported BAS sub-facet scores for reward interest and reward responsivity compared to healthy controls (Wilson et al., 2019).

The relationship between personality involving high levels of BIS sensitivity and restrictive disordered eating in anorexia, reported by Wilson et al. (2019), resonates with a theory of anorexia of the restrictive eating subtype. This theory suggests that conflict exists between a physiological drive to approach food and the conscious drive to restrict food intake to facilitate weight loss. This conflict would trigger anxiety and maintain the vicious cycle of the disorder (Frank, DeGuzman, \& Shott, 2019). This theory of anorexia can be accommodated within the perspective on personality proposed by rRST (Gray \& McNaughton, 2000). In rRST 
the drive to approach food would rely on BAS activation, the drive to avoid food would rely on FFFS activation, and the conflict induced anxiety would reflect BIS activation.

It has also been shown that elevated subclinical disordered eating relates to greater FFFS sensitivity, reduced overall BAS sensitivity, and also to low self-reported trait mindfulness (Wilson \& O'Connor, 2017). The term mindfulness refers to the increased awareness of thoughts, feelings, and body-sensations (Baer et al., 2008). Low trait mindfulness, in turn, relates to a range of restrictive and bulimic disordered eating behaviors (Lavender, Gratz, \& Tull, 2011; Lavender, Jardin, \& Anderson, 2009). Trait mindfulness correlates positively with self-reported AC, and both low trait mindfulness, and low AC scores predict elevated trait anxiety (Walsh et al., 2009). Whereas the term mindfulness refers to a broad range of thoughts, feelings, and sensations (Baer et al., 2008), the term attentional control refers to the explicit ability to focus and shift attention when required (Derryberry \& Reed, 2002).

There is a substantial genetic overlap concerning AC ability and trait anxiety (Gagne et al., 2017). For example, Attentional Control Theory (Eysenck, Derakshan, Santos, \& Calvo, 2007) suggests that AC impairments in elevated trait anxiety predict increased cognitive interference, which is further exacerbated if the distracting stimuli are threat-related. However, some participants with elevated trait anxiety may be able to inhibit threat-related stimulation if they have sufficient AC abilities (Derryberry \& Reed, 2002).

A combination of high trait anxiety and low AC predicts difficulties in inhibiting the processing of threat-related stimuli, relative to other combinations of trait anxiety and AC (Derryberry \& Reed, 2002). According to rRST, trait anxiety is generated by the BIS (Gray \& McNaughton, 2000). Disordered eating relates to thoughts of weight gain (Pereira \& Alvarenga, 2007). One can theorize that these thoughts may be experienced as threat-related stimuli which individuals with elevated BIS sensitivity and poor AC cannot inhibit, which would likely elevate disordered eating. However, it is possible that high BIS individuals with sufficient $\mathrm{AC}$ are able to inhibit these disordered eating related thoughts. Thus, the anxiety component of personality, and variability in AC abilities may combine to affect the likelihood of restrictive disordered eating.

Cognitive studies show that attentional shifting is impaired in the eating disorders (Roberts et al., 2007). However, it is unknown how self-reported AC predicts disordered eating. It is also unknown how the personality construct of BIS sensitivity interacts with the cognitive construct of AC to predict disordered eating. It is important to understand the relationship between disordered eating, BIS, and $\mathrm{AC}$, as it has been proposed that future experimental interventions that increase AC might reduce disordered eating relevant cognitions in anorexia of the restrictive eating subtype (Mercado et al., 2020).

\section{The present study}

The present study tests how self-reported BIS, BAS, FFFS, $\mathrm{AC}$, and trait mindfulness predict restrictive disordered eat- ing. Based on the above we predicted that correlation/regression analyses of these variables in the form of continuous data will show that elevated restrictive disordered eating relates to increased BIS and FFFS sensitivity, reduced AC, and reduced trait mindfulness.

We planned to conduct categorical analyses to explore whether high BIS and low AC predicts elevated restrictive disordered eating (compared to other combinations of BIS and $\mathrm{AC}$ ), and whether low BIS and high AC offer any protective effect against restrictive disordered eating (compared to other combinations of BIS and AC). This was inspired by the categorical trait anxiety and AC analysis by Derryberry and Reed (2002). We also used a further categorical analysis to test the frequency of participants with different combinations of BIS and AC in high and low restrictive disordered eating groups.

\section{METHOD}

\section{Participants}

The Department of Psychology, Sociology and Politics at Sheffield Hallam University granted ethical approval for this study. In line with the declaration of Helsinki, participants gave informed consent and had the right to withdraw. Participants were recruited via social media to participate in an online survey about personality and eating behaviors. The survey was conducted between 4th February and 25th March in the year 2021, amidst the COVID-19 pandemic. No financial incentive was offered. The survey was delivered using the Qualtrics ${ }^{1}$ platform.

We based our sample size on an approximate power calculation based on Wilson and O'Connor (2017). They reported zero order correlations between disordered eating and FFFS, BAS, and mindfulness, which ranged through $r$ $=0.15, r=-0.19$, and $r=-0.29$, respectively. We used the G-Power package (Faul, Erdfelder, Buchner, \& Lang, 2009) and determined that the smaller correlation of $r=0.15$ (at $p$ $=0.05$; providing $80 \%$ power; 2 -tailed) would require a minimum sample size of 343. Initially, 952 adult volunteers began the survey. After removing participants who submitted incomplete responses there were 641 participants remaining (166 males; mean age $=29.7 ; S D=13.8)$. Of the 641 participants, 302 were students (10 received course credit from the host institution).

The survey also screened participants for any previous history of psychiatric conditions; thus we created two groups of participants to enable us to control for this variable: a psychiatric group and a healthy group. The psychiatric group contained participants reporting a previous diagnosis of a mental health disorder $(N=160)$, neurological disorder $(N=4)$, developmental disorder $(N=2)$, or more than one of the disorders $(N=11)$. The psychiatric subsample size was thus 177 (26 males; mean age $=26.4 ; S D=11.2$ ). Of the 177 participants, 101 were students (4 received course credit from the host institution). The healthy subsample size was thus 464 (140 males; mean age $=31 ; S D=14.5)$. Of the 464 participants, 201 participants were students (6 received course credit from the host institution).

\footnotetext{
${ }^{1}$ The Qualtrics platform is an online survey tool where researchers can create and distribute self-report surveys.
} 
The survey did not ask respondents to specify their psychiatric disorders, so as to avoid any potential discomfort to the participants. Therefore, the classification of all those reporting a previous diagnosis of a mental health disorder into one group likely combines several types of disorder. However, contemporary psychiatry views all mental disorders as being highly comorbid, and views a person's liability to, the persistence of, and severity of, mental disorders as arising from individual differences in a common underlying ' $p$ ' factor, or psychopathology factor (Caspi \& Moffitt, 2018). We did not exclude participants with neurological or developmental disorders from the predominantly mental health disorder populated psychiatric group, as they were so few.

\section{Measures and procedure}

\section{Personality}

Participants were asked to give personality self-ratings using the 22-item Reinforcement Sensitivity Personality Questionnaire short version (RST-PQ-S; Vecchione \& Corr, 2020). In this questionnaire participants viewed a list of statements about everyday feelings and behaviors, and were asked to rate how accurately each statement describes them in general. They responded using a 4-point Likert scale ranging through not at all, slightly, moderately, and highly. The RST-PQ-S measures BIS sensitivity with 5 items (for example, It's difficult to get some things out of my mind), FFFS sensitivity with 5 items (for example, I would instantly freeze if I opened the door to find a stranger in the house), and BAS sensitivity with 12 items. The BAS scale is divided into four separate subscales. The BAS reward-interest (BAS-RI) subscale contains 3 items (for example, I get carried away by new projects), the BAS goal-drive persistence (BAS-GDP) subscale contains 3 items (for example, I am very persistent in achieving my goals), the BAS reward reactivity (BAS-RR) subscale contains 3 items (for example, good news makes me feel overjoyed), and the BAS impulsivity (BAS-I) subscale contains 3 items (for example, I find myself doing things on the spur of the moment). None of the RST-PQ-S items are reverse scored. Higher scores represent greater levels of each reinforcement sensitivity. The total sample mean BIS score was $14.2(95 \%$ $C I=13.4-13.9$, Cronbach's Alpha $(\alpha)=0.71)$. The total sample mean FFFS score was 11.8 (95\% CI =14-14.4, $\alpha=$ 0.56). The total sample mean BAS subscale scores were: BAS-RI: 7.3 (95\% CI =7.1-7.5, $\alpha=0.70)$; BAS-GDP: 9.1 (95\% CI = 8.9-9.3, $\alpha=0.81)$; BAS-RR: 9.2 (95\% CI =9.19.4, $\alpha=0.61)$; and BAS-I: $7.0(95 \% C I=6.8-7.2, \alpha=0.58)$.

\section{Eating attitudes}

We specifically measured restrictive disordered eating using the 8-item eating attitudes test (EAT-8; Richter et al., 2016)). Participants indicated if they agreed with the 8 statements (for example, I am preoccupied with a desire to be thinner; I feel extremely guilty after eating) using a two choice format: I agree somewhat (coded as 1) or I disagree somewhat (coded as 0 ). Higher scores indicate elevated restrictive subtype anorexia symptoms. The total sample mean score was 4.3 (95\% $C I=4.1-4.5, \alpha=0.83)$. EAT-8 scores were not skewed, were distributed through the whole scoring range, and the highest score was reported by 90 participants.

\section{General eating disorder symptom screening}

We also used the 5-item SCOFF screening measure (Morgan et al., 1999) as a supplementary general measure of binging, purging, and restrictive eating behaviors. Participants answered questions using a yes or no response format (for example, do you make yourself sick [vomit] because you feel uncomfortably full? Do you believe yourself to be fat when others say you are thin?), and higher scores indicated worse symptoms. In the present study the total sample mean score was $1.4(95 \% C I=1.3-1.5, \alpha=0.62)$.

\section{Attentional control}

We used the 20-item Attentional Control Scale (ACS; Derryberry \& Reed, 2002) to measure individual differences in self-reported attentional control. Participants indicated how often they experience effects of attention described in 20 statements which relate to attentional focusing abilities (for example, my concentration is good even if there is music in the room around me), and attentional shifting abilities (for example, I can quickly switch from one task to another). Participants responded using a 4 point Likert scale ranging through almost never, sometimes, often, and always. Higher scores indicated more efficient AC (11 of the items were reverse scored). The total sample mean score was $51.1(95 \%$ $C I=50.5-51.8, \alpha=0.85)$.

\section{Mindfulness}

We used the 15-item Five Facet Mindfulness Questionnaire (FFMQ-15; Baer et al., 2008) to measure trait mindfulness. Participants were required to use a 5 point Likert scale to indicate how true statements are of them (for example, I pay attention to sensations, such as the wind in my hair or sun on my face; I notice how foods and drinks affect my thoughts, bodily sensations, and emotions). The 5 point Likert scale ranged through never or very rarely true, rarely true, sometimes true, often true, and very often or always true (7 items were reverse scored). The total sample mean score was $46.1(95 \% C I=45.5-46.6, \alpha=0.71)$. Higher scores indicate higher trait mindfulness.

\section{Body weight/shape concern}

The COVID-19 pandemic, associated lockdown restrictions, and media coverage concerning weight gain will have likely exacerbated negative thoughts about body weight and/or shape. This will likely exacerbate restrictive disordered eating (Rodgers et al., 2020). Therefore we created an exploratory pandemic-related body weight/shape concern (PBWSC) question, for use in a hierarchical regression analysis. We asked participants: "during the COVID19 /lockdown periods have you been less or more interested in your own body weight or body shape than usual?" This question was scored on a 3 point scale: less than usual $=1$; same as usual $=2$; more than usual $=3$ (total sample mean score $=2.4 ; 95 \% C I=2.4-2.5$ ).

\section{Analyses}

Our preliminary correlational analysis illustrated how the predictor variables related to one another. This was followed by a hierarchical multiple regression to illustrate how the combination of our predictor variables predicted EAT-8 scores, whilst holding sex, participant group (healthy versus 

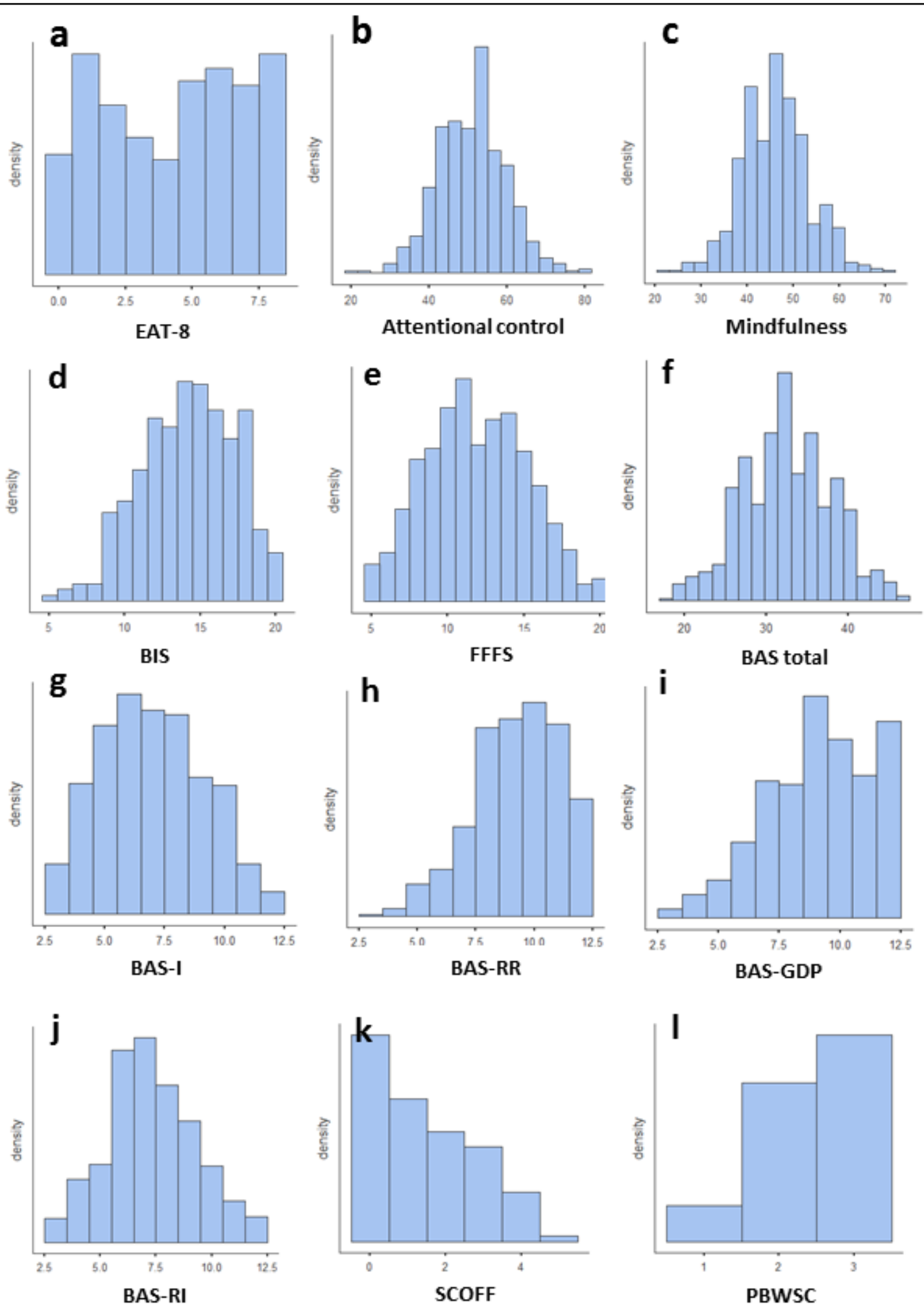

Figure 1. The data distributions for the EAT-8 (panel a), attentional control (panel b), mindfulness (panel c), BIS (panel d), FFFS (panel e), BAS total (panel f), BAS-I (panel g), BAS-RR (panel h), BAS-GDP (panel i), BAS-RI (panel j), SCOFF (panel k), and pandemic-related body/weight shape concern scores (PBWSC; panel 1). 
Table 1. Spearman's Rho correlations between the self-report measures for the whole sample

\begin{tabular}{|c|c|c|c|c|c|c|c|}
\hline & 1 & 2 & 3 & 4 & 5 & 6 & 7 \\
\hline 1. EAT 8 & - & & & & & & \\
\hline 2. SCOFF & $0.713 * * *$ & - & & & & & \\
\hline 3. BIS & $0.348 * * *$ & $0.360 * * *$ & - & & & & \\
\hline 4. BAS & 0.044 & 0.009 & $0.083^{*}$ & - & & & \\
\hline 5. FFFS & $0.196 * * *$ & $0.136 * * *$ & $0.233 * * *$ & 0.043 & - & & \\
\hline 6. ACS & $-0.292 * * *$ & $-0.272 * * *$ & $-0.399 * * *$ & $0.101 *$ & $-0.253 * * *$ & - & \\
\hline 7. FFMQ & $-0.253 * * *$ & $-0.309 * * *$ & $-0.543 * * *$ & $0.116^{* *}$ & $-0.152 * * *$ & $0.502 * * *$ & - \\
\hline 8. PBWSC & $0.279 * * *$ & $0.235 * * *$ & $0.181 * * *$ & $0.092 *$ & $0.082^{*}$ & $-0.130 * * *$ & $-0.130 * * *$ \\
\hline
\end{tabular}

Note: $* \mathrm{p}<.05, * * \mathrm{p}<.01,{ }^{* * *} \mathrm{p}<.001$

psychiatric), and the single item pandemic-related body weight/shape concern question constant.

Because hierarchical multiple regression may illustrate the cumulative and individual associations between our predictor variables and EAT- 8 scores, it does not reveal how different combinations of BIS and AC affect EAT-8 scores, or reveal how many participants with different combinations of BIS and AC appear in high or low levels of EAT-8 measured restrictive disordered eating. Therefore, we also used a series of categorical analyses based upon median splits in order to test how the different combinations of BIS and AC predict EAT-8 scores. The use of median splits is justifiable if the analysis is used to clarify effects found in an initial analysis, which included the same set of variables as continuous variables (DeCostera, Galluccib \& Iselinc, 2011).

We used a categorical analysis based upon median splits to create four different BIS and AC combination groups. This first categorical analysis tested our hypothesis that the combination of high BIS and low AC will predict increased EAT-8 scores (which remained as a continuous variable) relative to other combinations of BIS and AC. We used a second categorical analysis to estimate the frequency of participants with different combinations of BIS and AC in high and low restrictive disordered eating groups (that were derived from a further median split on the EAT- 8 variable).

We note here that we are not implying that the median splits used to delineate high and low BIS, AC or EAT-8 groups represent screening, diagnostic, or epidemiological cut-off scores. In our analysis and in the discussion we use the terms high and low to refer to participants above or below the median scores within the data distributions in the present study. Richter et al. (2016) provide potential (but seemingly debated) screening cut-off scores for the EAT-8 measure, but these are surprisingly low, and as such $72 \%$ of our sample from the general population would be in the high disordered eating category. Figure 1 (panel a) shows the distribution of EAT-8 scores in the present study, and it shows that a median split is more appropriate for an analysis of the relationship between personality and the extent of restrictive disordered eating behaviors. The use of a median split on the EAT-8 variable also matches the (necessary) use of median splits on the BIS and AC variables (that have no suggested screening cut-off scores).

We also reiterate here that we are interested in how the co-occurrence of BIS and AC affect restrictive disordered eating. Therefore, we are not hypothesizing that AC mediates any BIS effects on EAT-8 scores, or that BIS mediates any AC effects on EAT-8 scores. We have no justification for implying that either variable affected our participants before the other variable affected them. Our cross-sectional correlational design prohibits proving any mediation effects between variables: such an analysis could create the temporal illusion that the effect of one of the BIS or AC variables predated the effect of the other (Roe, 2012).

\section{RESULTS}

\section{Continuous variable analyses}

Figure 1 gives the data distributions for the self-report measures in 12 panels. Most of the scoring distributions were not badly skewed with the exception of the supplementary SCOFF measure and the exploratory pandemic-related body/weight shape concern (PBWSC) question. Despite this, we used non-parametric correlations for all comparisons in an initial analysis. Table 1 contains the zero-order Spearman's Rho correlations between the variables for the whole sample of 641 participants. This analysis confirmed that our primary specific measure of restrictive disordered eating (the EAT-8) was strongly positively correlated with the SCOFF, our supplementary general measure of bingepurge and restrictive disordered eating behavior. This analysis also confirmed that BIS and AC were negatively correlated as were BIS and mindfulness (FFMQ). EAT-8 scores were positively correlated with BIS, FFFS, and PBWSC, and were negatively correlated with $\mathrm{AC}$ and trait mindfulness (FFMQ). As the total BAS score was not significantly correlated with EAT-8 we also probed how the 4 BAS subscales related to EAT-8 scores. EAT- 8 was weakly positively correlated with BAS-I ( $r \mathrm{~s}=0.12, p=0.002)$, but it was not meaningfully correlated with BAS-RR, BAS-RI or BAS-GDP (all $r \mathrm{~s}<0.08$, all $p \mathrm{~s}>0.05$ ).

There are some revised factorial structures to the Attentional Control Scale (ACS) that contain separate attentional focussing and attentional shifting subscales (Judah, Grant, Mills \& Lechner, 2013; Olafsson et al., 2011). We confirmed that the Spearman's Rho correlation values between EAT-8 scores and all four ACS subscales were similar in strength (all $r \mathrm{~s}>-0.25$, all $p \mathrm{~s}<0.001$ ). As restrictive disor- 
Table 2. Hierarchical multiple regression of participant group (psychiatric/healthy: denoted as P-group), sex, pandemicrelated body weight/shape concern (denoted as PBWSC), mindfulness, ACS, and reinforcement sensitivity scores onto EAT-8 scores

\begin{tabular}{llllc}
\hline & $B$ & SE & Beta & $p$ \\
\hline Model 1 & & & & \\
P-group & 1.0 & 0.22 & 0.17 & $<0.001$ \\
Sex & 1.2 & 0.22 & 0.20 & $<0.001$ \\
PBWSC & 0.96 & 0.15 & 0.24 & $<0.001$
\end{tabular}

\section{Model 2}

\begin{tabular}{lrrrr} 
P-group & 0.54 & 0.22 & 0.09 & 0.014 \\
Sex & 0.84 & 0.23 & 0.14 & $<0.001$ \\
PBWSC & 0.78 & 0.14 & 0.19 & $<0.001$ \\
Mindfulness & -0.01 & 0.02 & -0.02 & 0.613 \\
ACS & -0.03 & 0.01 & -0.11 & 0.009 \\
BIS & 0.16 & 0.04 & 0.18 & $<0.001$ \\
FFFS & 0.05 & 0.03 & 0.06 & 0.129 \\
BAS-I & 0.05 & 0.04 & 0.04 & 0.248 \\
\hline
\end{tabular}

dered eating was clearly negatively correlated with both attentional shifting and attentional focusing we used the total ACS scores in the rest of our analyses.

Table 2 gives the results of the hierarchical multiple regression of participant group (P-group: psychiatric/healthy), sex, pandemic-related body weight/shape concern, ACS, mindfulness (FFMQ), BIS, FFFS and BAS-I onto EAT-8 scores. The residuals were normally distributed, and multicollinearity was very low. Model 1 was significant $(R=$ $0.383 ; R^{2}=0.147 ;$ adjusted $R^{2}=0.143 ; F(3,637)=36.6 ; p<$ 0.001 ), and it explained $14.3 \%$ of the variance in EAT- 8 scores. Table 2 shows that in Model 1 sex significantly predicted EAT-8 scores, with females (mean $=4.3 ; S D=2.6$ ) scoring higher than males (mean $=3.0 ; S D=2.3$ ). In Model 1 , pandemic-related body shape/weight concern and participant group also significantly predicted EAT-8 scores. The psychiatric subsample $($ mean $=5.2 ; S D=2.5)$ scored higher than the healthy subsample (mean $=3.9 ; S D=2.6)$. Model 2 was also significant $\left(R=0.474 ; R^{2}=0.225\right.$; adjusted $R^{2}=$ $0.215 ; F(8,632)=22.9 ; p<0.001)$. Model 2 explained an additional $7.8 \%$ of the variance in EAT- 8 scores $\left(R^{2}\right.$ change $=0.078 ; F$ change $=12.7, p<0.001)$. Notably, when holding participant group, sex, and pandemic-related body weight/shape concern constant, Model 2 shows that BIS was a prominent positive predictor of EAT- 8 scores, whereas $\mathrm{AC}$ was a prominent negative predictor of EAT-8 scores.

\section{Categorical variable analyses}

In the following, we applied categorical analysis to test our hypothesis that the combination of high BIS and low AC predicts increased restrictive disordered eating more strongly than the other BIS-AC combinations. For the whole sample of 641 participants the median score for BIS was 14, and the median score for AC was 51. Using median splits, we divided the whole sample into four groups: high BIS and low AC (whole sample $N=195$ ), high BIS and high AC (whole sample $N=106$ ), low BIS and low AC (whole sample $N=75$ ), and low BIS and high AC (whole sample $N=$ 171). Participants with either a median BIS or AC score (or both) were excluded, leading to a total BIS-AC sample size of 547.

Before conducting this analysis we noted that MannWhitney tests showed that BIS scores were higher in the psychiatric subsample than the healthy subsample $(U=$ 26155.0, $Z=7.1, p<0.001)$, whereas trait mindfulness scores $(U=27548.0, Z=6.5, p<0.001)$, and AC scores $(U$ $=28691.5, Z=5.9, p<0.001)$ were lower in the psychiatric subsample than the healthy subsample. By contrast, the two groups did not differ in FFFS $(U=39712.0, Z=0.6, p=$ $0.517)$, BAS $(U=39570, Z=0.7, p=0.475)$, or the exploratory pandemic-related body weight/shape concern question ( $U=38610, Z=1.3, p=0.191)$. Considering the group differences in BIS and AC, we conducted the analyses of how the different combinations of BIS and AC predict restrictive disordered eating separately for the healthy subsample and the psychiatric subsample.

Figure 2 shows the distribution of EAT- 8 scores in each of the BIS-AC groups, separated by the healthy (panel a) and psychiatric (panel b) subsamples. For the healthy subsample $(N=394)$, a one-way Kruskal-Wallis test showed that EAT-8 scores differed between the four BIS-AC groups $\left(\chi^{2}(3)=45.9, p<0.001\right)$. Mann-Whitney tests showed that the high BIS low AC group reported higher EAT-8 scores than the low BIS low AC group $(U=2151.0, Z=3.6, p<$ $0.001)$, the high BIS high AC group $(U=3214.5, Z=2.6, p$ $=0.009)$, and the low BIS high AC group $(U=4238.5, Z=$ $6.7, p<0.001)$. The high BIS high AC group reported higher EAT-8 scores than the low BIS high AC group $(U=4178.0$, $Z=3.3, p=0.001)$. There were no other significant EAT-8 score differences between the healthy subsample BIS-AC groups.

For the psychiatric subsample $(N=153)$ a one-way Kruskal-Wallis test showed that EAT-8 scores differed between the four BIS-AC groups $\left(\chi^{2}(3)=14.2, p=0.003\right)$. MannWhitney tests showed that the high BIS low AC group reported higher EAT-8 scores than the low BIS high AC group ( $U=441.0, Z=3.7, p<0.001)$, and the high BIS high AC group reported higher EAT-8 scores than the low BIS high AC group $(U=194.5, Z=2.3, p=0.020)$. There were no other significant EAT- 8 score differences between the psychiatric subsample BIS-AC groups.

We used a median split on the EAT-8 data to reveal how many participants with different combinations of BIS-AC appear in high or low levels of restrictive disordered eating. The exclusion of participants with a median EAT-8 score of 5 (in addition to those with median BIS and/or AC scores) left a subsample of 350 for the healthy group, and a subsample of 130 for the psychiatric group.

Table 3 shows the cross-tabulation of the observed counts for the combinations of EAT- 8 and BIS-AC scores for the healthy subsample and separately for the psychiatric subsample. We used two separate $4 \times 2 \chi^{2}$ tests to analyze the frequency of participants with the four different combinations of BIS-AC in the high and low EAT- 8 groups, in the separate healthy and psychiatric subsamples.

For the healthy subsample, there was a significant association between BIS-AC combination and EAT-8 group 


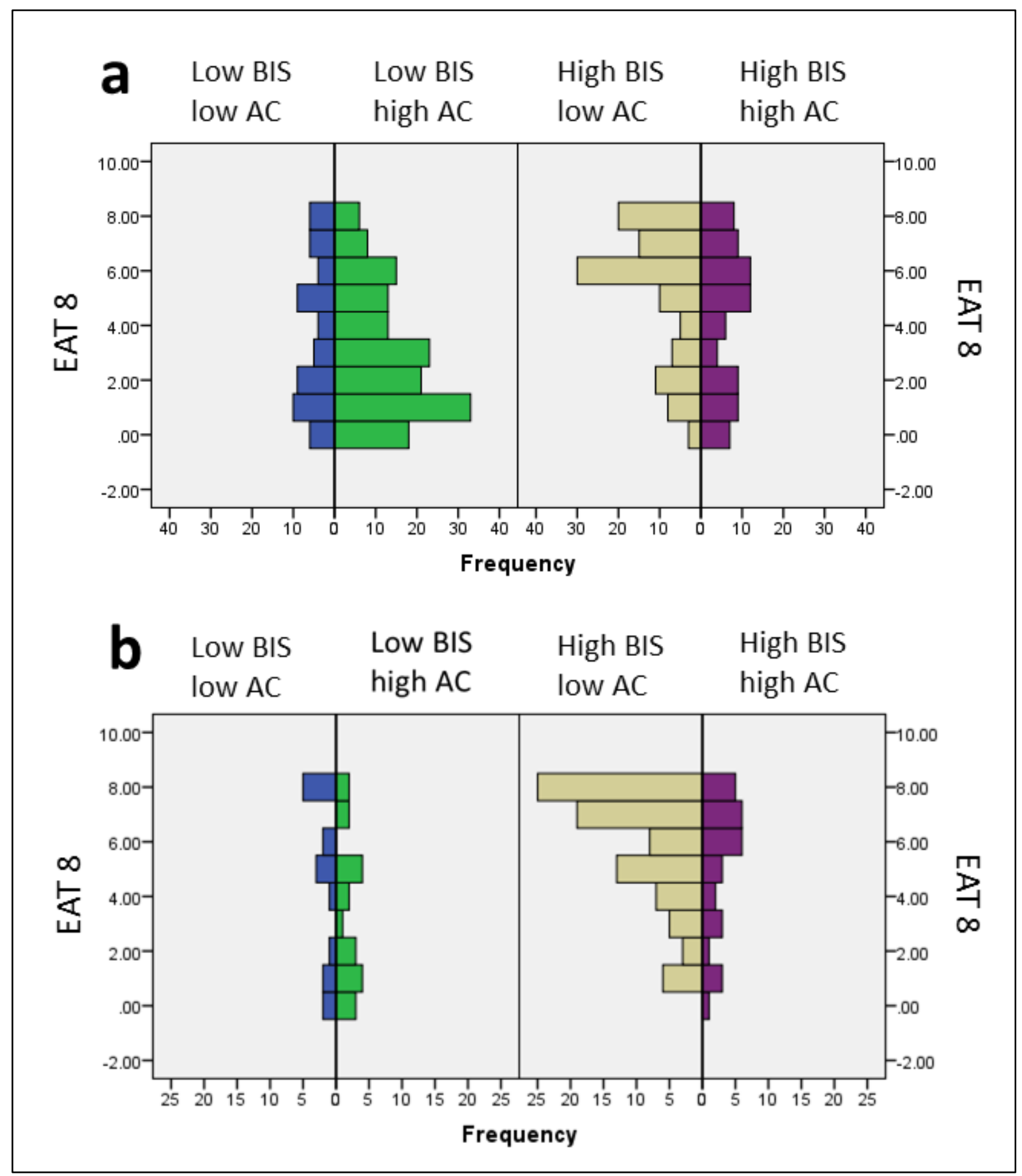

Figure 2. The distribution of EAT-8 scores in each of the BIS-AC groups, separated by the healthy (panel a) and psychiatric (panel b) subsamples.

$\left(\chi^{2}(3)=49.6, p<0.001\right)$. Cramer's $V(0.38)$ suggested that $14.4 \%$ of the variation in EAT- 8 group classification could be explained by the BIS-AC combinations. For the psychiatric subsample there was a significant association between BIS-AC combination and EAT- 8 group $\left(\chi^{2}(3)=13.6, p=\right.$ $0.003)$. Cramer's $V(0.32)$ suggested that $10.2 \%$ of the variation in EAT-8 group classification could be explained by the BIS-AC combinations.
Table 3 shows the number of participants with each BISAC combination in the low and high EAT-8 groups, for the healthy group and psychiatric group. In correspondence to the numbers of participants in Table 3, Table 4 gives the percentages of participants in the low and high EAT-8 groups reporting each of the four BIS-AC combinations. Table 4 shows that in the healthy subsample $51.2 \%$ of the participants in the low EAT-8 group reported low BIS and high 
Table 3. The cross-tabulation of the observed counts for the EAT-8 and BIS-AC groups for the psychiatric subsample and the healthy subsample

\begin{tabular}{lccccc}
\hline & & Low BIS, Low AC & Low BIS, High AC & High BIS, Low AC & High BIS, High AC \\
\hline \multirow{2}{*}{ Healthy subsample } & Low EAT-8 & 34 & 108 & 34 & 35 \\
& High EAT-8 & 16 & 29 & & 25 \\
\multirow{2}{*}{ Psychiatric subsample } & Low EAT-8 & 6 & 13 & 21 & 10 \\
& High EAT-8 & 7 & 4 & 52 & 17 \\
\hline
\end{tabular}

Table 4. The percentage of participants in the low and high EAT-8 groups with each of the four BIS-AC combinations

\begin{tabular}{|c|c|c|c|c|c|}
\hline & & Low BIS, Low AC & Low BIS, High AC & High BIS, Low AC & High BIS, High AC \\
\hline \multirow{2}{*}{ Healthy subsample } & Low EAT-8 & $16.1 \%$ & $51.2 \%$ & $16.1 \%$ & $16.6 \%$ \\
\hline & High EAT-8 & $11.5 \%$ & $20.9 \%$ & $46.8 \%$ & $20.9 \%$ \\
\hline \multirow{2}{*}{ Psychiatric subsample } & Low EAT-8 & $12.0 \%$ & $26.0 \%$ & $42.0 \%$ & $20.0 \%$ \\
\hline & High EAT-8 & $8.8 \%$ & $5.0 \%$ & $65.0 \%$ & $21.3 \%$ \\
\hline
\end{tabular}

Table 5. The percentage of participants in each of the four BIS-AC groups with either low or high EAT- 8 scores

\begin{tabular}{lccccc}
\hline & & Low BIS, Low AC & Low BIS, High AC & High BIS, Low AC & High BIS, High AC \\
\hline \multirow{2}{*}{ Healthy subsample } & Low EAT-8 & $68.0 \%$ & $78.8 \%$ & $34.3 \%$ & $54.7 \%$ \\
& High EAT-8 & $32.0 \%$ & $21.2 \%$ & $65.7 \%$ & $45.3 \%$ \\
\multirow{2}{*}{ Psychiatric subsample } & & & & \\
& Low EAT-8 & $46.2 \%$ & $76.5 \%$ & $28.8 \%$ & $37.0 \%$ \\
& High EAT-8 & $53.8 \%$ & $23.5 \%$ & $71.2 \%$ & $63.0 \%$ \\
\hline
\end{tabular}

AC. By contrast, in the psychiatric subsample, $26 \%$ of participants in the low EAT-8 group reported low BIS and high AC. It is noteworthy that in the healthy subsample, $20.9 \%$ of the participants in the high EAT-8 group reported low BIS and high AC, whereas in the psychiatric subsample, 5\% of the participants in the high EAT-8 group reported low BIS and high AC. Table 4 also shows that $46.8 \%$ of the participants in the high EAT- 8 group in the healthy subsample reported high BIS and low AC, and $65 \%$ of the participants in the high EAT-8 group in the psychiatric subsample reported high BIS and low AC.

Table 4 contains an interpretation of the cross-tabulation data from Table 3 where the counts in the cells are expressed as the percentage of participants in the high and low EAT-8 groups who reported each of the four BIS-AC combinations. However, the cross-tabulation data from Table 3 can also be interpreted in a different way. The counts in the cells can also be expressed as the percentages of participants in each of the four BIS-AC groups who reported either low or high EAT-8 scores. This different interpretation of the data is presented in Table 5. Table 5 clearly shows that in both the healthy and psychiatric subsamples over $76 \%$ of participants reporting low BIS and high AC also reported low EAT-8 scores, and that over $65 \%$ of participants reporting high BIS and low AC also reported high EAT-8 scores.

\section{DISCUSSION}

Elevated EAT-8 measured restrictive disordered eating related to increased BIS sensitivity and FFFS sensitivity, consistent with Wilson et al. (2019). In the present study, FFFS was, however, not particularly prominent in the regression analysis. Research using BIS measures, inspired by an earlier version of RST (Gray, 1982), have also found that elevated disordered eating (Hasking, 2006), and anorexia and bulimia symptoms (Harrison, Treasure, \& Smillie, 2011) relate to elevated BIS scores.

The combination of FFFS sensitivity and BIS sensitivity contributes to the more general personality trait of neuroticism (Smillie, 2008). High neuroticism is a risk factor for disordered eating (Miller et al., 2006). Elevated neuroticism relates to reduced AC capabilities (Meesters, Muris, \& van Rooijen, 2007), and to reduced trait mindfulness (Feltman, Robinson, \& Ode, 2009). In the present study, lower levels of trait mindfulness predicted increased disordered eating, which is consistent with previous research (Lavender et al., 2011, 2009; Wilson \& O'Connor, 2017). In the present study, however, the Beta values in the regression analysis show that AC predicted EAT-8 restrictive disordered eating more prominently than trait mindfulness predicted EAT- 8 restrictive disordered eating. When holding sex and the sin- 
gle item pandemic-related body weight/shape concern question constant, BIS was a prominent positive predictor of EAT- 8 scores, and AC was a prominent negative predictor of EAT-8 scores. This shows that both personality and AC processes are related to restrictive disordered eating.

Categorical analyses showed that when considering the proportion of participants in each of the four BIS-AC groups with either low or high EAT-8 scores, over $65 \%$ of participants reporting high BIS and low AC also reported high EAT- 8 scores in both the healthy and psychiatric groups. Moreover, over $75 \%$ of participants reporting low BIS and high AC reported low EAT-8 scores in both the healthy and psychiatric groups. This suggests that a high BIS personality individual with low AC ability might be at risk for elevated restrictive disordered eating. By contrast, a low BIS personality individual with high AC ability might be less susceptible to this type of eating pathology. When interpreting the same data from the perspective of the EAT-8 groupings, however, it is notable that not all participants with high EAT-8 scores displayed the pattern of high BIS and low AC, and not all of the participants with low EAT-8 scores displayed the pattern of low BIS high AC. Our data show that for some people a combination of low BIS and high AC might be a protective factor against the development of disordered eating, but this is not the case for everybody.

A predominantly rRST inspired exposition of our data would suggest that in restrictive disordered eating the fear of weight gain, and the conflicting need to eat, would likely increase BIS activity (and thus anxiety) in individuals already phenotypically high in BIS, as the BAS and FFFS would be co-activated. One can theorize that when eating is likely to occur, elevated BIS activity will often facilitate FFFS congruent avoidance responses if a person suffers from restrictive disordered eating. A simple extension to this account would suggest that in some people elevated BIS sensitivity co-occurs with impoverished AC abilities. Impaired AC may contribute to disordered eating behaviors by exacerbating self-referential negative thought processes. For example, attentional shifting impairments may exacerbate difficulties in shifting thoughts away from possible weight gain and/or body shape and thus reduce attentional focusing on food consumption and health requirements. This explanation resonates with the Attentional Control Theory of anxiety (Eysenck et al., 2007) which suggests that AC impairments in elevated trait anxiety exacerbate threatrelated distraction.

We note here that a healthy diet is associated with better executive functioning compared to an unhealthy diet (Cohen et al., 2016). Thus, it is also plausible that poor nutrition resulting from disordered eating can impair AC, which may further exacerbate BIS activity in those with a high BIS phenotype. However, it is entirely possible that a reciprocal relationship between elevated disordered eating and the combination of high BIS and low AC could develop. In this case, elevated disordered eating and the combination of high BIS and low AC would mutually reinforce one another. This might explain why in some people disordered eating behaviors do not easily dissipate.

Our results should be interesting to personality researchers, but should also be of use to clinicians and/or medical practitioners who are developing novel eating disorder in- terventions. It may be useful to tailor the design of such interventions to suit individual participants BIS and/or AC profiles. A recent commentary has suggested that attentional bias modification (ABM) could be used to treat restrictive disordered eating behavior (Mercado et al., 2020). Mercado et al. discuss two mechanistic models. The first model of $\mathrm{ABM}$ involves increasing general $\mathrm{AC}$ in order to dampen disordered eating relevant thoughts. The second model of ABM involves using exposure to food stimuli to facilitate a re-evaluation of food, in a way that reduces avoidance and fear responses. Mercado et al. do not discuss rRST (Gray \& McNaughton, 2000) but their second model clearly implies there is a need to reduce FFFS activity in restrictive disordered eating. The present study has revealed a general impairment in $\mathrm{AC}$ (that is not specific to eating related stimuli) in a large proportion of participants reporting restrictive disordered eating. Therefore a future experimental intervention study might test the utility of the first model of ABM (Mercado et al., 2020) as an initial attention training mechanism for participants with low (but not high) self-reported AC. This could be followed with the second model of ABM specifically involving food stimuli, as described by Mercado et al. (2020). However, elevated pre-treatment BIS sensitivity can predict higher post-treatment anxiety levels following cognitive behavioral interventions for anxiety (Ly, 2011). Considering that participants with high BIS levels might be harder to treat, and/or take longer to treat (Ly, 2011), it may be worth assessing BIS levels prior to determining the length of future experimental eating disorder interventions.

\section{Limitations}

Despite the utility of our data, our study has limitations. Self-report measures were used to assess the manifestations of personality produced by the theoretical brain-behavioral systems described in rRST (Gray \& McNaughton, 2000). Future research may benefit from using behavioral and/or physiological measures of the reactivity of these brain-behavioral systems in disordered eating. Moreover, although we were able to divide our sample into a general psychiatric subsample and a healthy subsample, a replication study that specifically recruits eating disorder patients would be useful. As discussed above, our high and low EAT-8, BIS, and AC groups are not diagnostic groups, they are groups based upon median splits internal to the data distributions in the present study. Finally, our cross-sectional correlational design prohibits proving any causal links between variables. Future longitudinal research examining how the combination of BIS sensitivity and AC predicts the development of disordered eating would therefore be useful.

\section{Conclusion}

Both personality and individual differences in self-reported cognitive ability such as AC are implicated in restrictive disordered eating. Variations in the personality construct of BIS sensitivity and the cognitive construct of AC seemed to be the most prominent predictors of restrictive disordered eating. We provide novel evidence that different combinations of BIS and AC ability can predict the severity of re- 
strictive disordered eating behaviors. However, differential combinations of self-reported BIS and AC do not predict restrictive disordered eating in the same way for everybody.

\section{REFERENCES}

Baer, R. A., Smith, G. T., Lykins, E., Button, D., Krietemeyer, J., Sauer, S., Walsh, E., Duggan, D., \& Williams, J. M. G. (2008). Construct validity of the Five Facet Mindfulness Questionnaire in Meditating and Nonmeditating Samples. Assessment, 15, 329342.

Bijttebier, P., Beck, I., Claes, L., \& Vandereycken, W. (2009). Gray's Reinforcement Sensitivity Theory as a framework for research on personality-psychopathology associations. Clinical Psychology Review, 29, 421-430.

Caspi, A., \& Moffitt, T. E. (2018). All for one and one for all: Mental disorders in one dimension. The American Journal of Psychiatry, 175, 831-844.

Cohen, J. F. W., Gorski, M. T., Gruber, S. A., Kurdzie, L. B. F., \& Rimm, E. B. (2016). The effect of healthy dietary consumption on executive cognitive functioning in children and adolescents: a systematic review. British Journal of Nutrition, 116, 989-1000.

DeCostera, J., Galluccib, M., \& Iselinc, A. M. R. (2011). Best Practices for Using Median Splits, Artificial Categorization, and their Continuous Alternatives. Journal of Experimental Psychopathology, 2, 197-209.

Derryberry, D., \& Reed, M. A. (2002). Anxiety related attentional biases and their regulation by attentional control. Journal of Abnormal Psychology, 111, 225-236.

Eysenck, M. W., Derakshan, N., Santos, R., \& Calvo, M. G. (2007). Anxiety and cognitive performance: Attentional control theory. Emotion, 2, 336-353.

Faul, F., Erdfelder, E., Buchner, A., \& Lang, A.-G. (2009). Statistical power analyses using G*Power 3.1: Tests for correlation and regression analyses. Behavior Research Methods, 41, 11491160.

Feltman, R., Robinson, M. D., \& Ode, S. (2009). Mindfulness as a moderator of neuroticism-outcome relations: A self-regulation perspective. Journal of Research in Personality, 43, 953-961.

Frank, G. K. W., DeGuzman, M. C., \& Shott, M. E. (2019). Motivation to eat and not to eat - The psycho-biological conflict in anorexia nervosa. Physiology \& Behavior, 206, 185-190.

Gagne, J. R., O’Sullivan, D. L., Schmidt, N. L., Spann, C. A., \& Goldsmith, H. H. (2017). The shared etiology of attentional control and anxiety: An Adolescent Twin Study. Journal of Research on Adolescence, 27, 122-138.

Gray. J. A. (1982). The Neuropsychology of Anxiety: An Enquiry into the Functions of the Septo-Hippocampal System. Oxford University Press, U.S.

Gray J. A., \& McNaughton, N. (2000). The Neuropsychology of Anxiety: An Enquiry into the Functions of the Septo-Hippocampal System. Oxford University Press.

Harrison, A., Treasure, J., \& Smillie, L. D. (2011). Approach and avoidance motivation in eating disorders. Psychiatry Research, 188, 396-401.

Hasking, A. P. (2006). Reinforcement sensitivity, coping, disordered eating and drinking behavior in adolescents. Personality and Individual Differences, 40, 677-688.

Judah, M. R., Grant, D. M., Mills, A. C., \& Lechner, W. V. (2013). Factor structure and validation of the attentional control scale. Cognition \& Emotion, 28, 433-451.

Kärkkäinen, U., Mustelin, L., Raevuori, A., Kaprio, J., \& KeskiRahkonen, A. (2018). Do Disordered Eating Behaviors Have Long-term Health-related Consequences? European Eating Disorders Review, 26, 22-28.
Lavender, J. M., Gratz, K. L., \& Tull, M. T. (2011). Exploring the relationship between facets of mindfulness and eating pathology in women. Cognitive Behavior Therapy, 40, 174-182.

Lavender, J. M., Jardin, B. F., \& Anderson, D. A. (2009). Bulimic symptoms in undergraduate men and women: Contributions of mindfulness and thought suppression. Eating Behaviors, 10, 228-231.

Ly, C. (2011). The relevance of reinforcement sensitivity theory to social anxiety and response to cognitive behavioral therapy for social anxiety disorder, Doctoral thesis, University of Tasmania.

Mercado, D., Schmidt, U., O'Daly, O, G., Campbell, L. C., \& Werthmann, J. (2020). Food related attention bias modification training for anorexia nervosa and it potential underpinning mechanisms. Journal of Eating Disorders, 8, 1-4.

Miller, J. L., Schmidt, L. A., Vaillancourt, T., McDougall, P., \& Laliberte, M. (2006). Neuroticism and introversion: A risky combination for disordered eating among a non-clinical sample of undergraduate women. Eating Behaviors, 7, 69-78.

Morgan J. F., Reid, F., \& Lacey, J. H. (1999). The SCOFF questionnaire: assessment of a new screening tool for eating disorders. BMJ. 319, 1467-1468.

Meesters, C., Muris, P., \& van Rooijen, B. (2007). Relations of Neuroticism and Attentional Control with Symptoms of Anxiety and Aggression in Non-Clinical Children. Journal of Psychopathology and Behavioral Assessment, 29, 149-158

Ólafsson, R. P., Smári, J. Guðmundsdóttir, F., Ólafsdóttir, G., Harðardóttir, H. .L., \& Einarsson, S. M. (2011). Self reported attentional control with the Attentional Control Scale: Factor structure and relationship with symptoms of anxiety and depression. Journal of Anxiety Disorders, 25, 777-782.

Pereira, R. F., \& Alvarenga, M. (2007). Disordered Eating: Identifying, Treating, Preventing, and Differentiating It from Eating Disorders. Diabetes Spectrum, 20, 141-148.

Richter, F., Strauss, B., Brähler, E., Altmann, U., \& Berger, U. (2016). Psychometric properties of a short version of the Eating Attitudes Test (EAT-8) in a German representative sample. Eating Behaviors, 21, 198-204.

Roberts, M., Tchanturia, K., Stahl, D., Southgate, L., \& Treasure, J. (2007). A systematic review and meta-analysis of set-shifting ability in eating disorders. Psychological Medicine, 37, 10751084.

Rodgers, R. F., Lombardo, C., Cerolini, S., Franko, D. L., Omori, M., Fuller-Tyszkiewicz, M., Linardon, J., Courtet, P., \& Guillaume, S. (2020). The impact of the COVID-19 pandemic on eating disorder risk and symptoms. International Journal of Eating Disorders, 53, 1166-1170.

Roe, R. A. (2012). What is wrong with mediators and moderators? The European Health Psychologist, 14, 4-9.

Smillie, L. D. (2008). What is Reinforcement Sensitivity? Neuroscience Paradigms for Approach-avoidance Process Theories of Personality. European Journal of Personality, 22, 359-384.

Vecchione, M. \& Corr, P. J. (2021). Development and Validation of a Short Version of the Reinforcement Sensitivity Theory of Personality Questionnaire (RST-PQ-S), Journal of Personality Assessment, 103, 535-546.

Walsh, J. J., Balint, M. G., Smolira SJ, D. R., Fredericksen, L. K., \& Madsen, S. (2009). Predicting individual differences in mindfulness: The role of trait anxiety, attachment anxiety and attentional control. Personality and Individual Differences, 46, 94-99.

Wilson, D., \& O'Connor, E. L. (2017). Mindfulness, personality and disordered eating. Personality and Individual Differences, $119,7-12$

Wilson, D. R., Loxton, N. J., O'Shannessy, D., Sheeran, N., \& Morgan, A. (2019). Similarities and differences in revised reinforcement sensitivities across eating disorder subtypes. Appetite, $133,70-76$ 